\title{
New insights into diabetes mellitus and its complications: a narrative review
}

\author{
Fei Hua \\ Department of Endocrinology, the Third Affiliated Hospital of Soochow University, Changzhou, China \\ Correspondence to: Prof. Fei Hua, MD, PhD. Department of Endocrinology, the Third Affiliated Hospital of Soochow University, Changzhou, China. \\ Email: czhuafei@vip.sina.com.
}

\begin{abstract}
Diabetes is a metabolic disorder accompanied by complications of multiple organs and systems. Diabetic nephropathy (DN) is one of the most prevalent lethal complications of diabetes. Although numerous biomarkers have be clarified for early diagnosis of DN, renal biopsy is still the gold standard. As a noninvasive imaging diagnostic method, blood oxygen level-dependent (BOLD) MRI can help understand the kidney oxygenation status and fibrosis process and monitor the efficacy of new drugs for DN via monitoring renal blood oxygen levels. Recent studies have shown that noncoding RNAs including microRNAs (miRNAs), long noncoding RNAs (lncRNAs) and circular RNAs (circRNAs) were all involved in the development of DN, which could be exploited as therapeutic strategy to control DN. Dyslipidemia is also a common complication of diabetes. Apolipoprotein $\mathrm{M}$ (apoM), as a novel apolipoprotein, may be related to the development and progression of diabetes, which need to further investigation. Obstructive sleep apnea (OSA) is another common complication of diabetes and is an independent risk factor for cardiovascular disease (CVD). At present, there is no simple, effective and rapid diagnostic method to early identification of OSA in patients with diabetes. A nomogram consisted of waist-to-hip ratio, smoking status, body mass index, serum uric acid, HOMA-IR and history of fatty liver might be an alternative method to early assess the risk of OSA.
\end{abstract}

Keywords: Diabetes mellitus (DM); diabetic nephropathy (DN); dyslipidemia; apolipoprotein M (apoM); obstructive sleep apnea (OSA)

Submitted Aug 06, 2020. Accepted for publication Dec 16, 2020.

doi: 10.21037/atm-20-7243

View this article at: http://dx.doi.org/10.21037/atm-20-7243

\section{Introduction}

Diabetes mellitus (DM), as a growing epidemic of bipolar disorder, affects near $5.6 \%$ of the world's population (1). Its global prevalence was about $8 \%$ in 2011 and is predicted to rise to $10 \%$ by 2030 (2). Likewise, its prevalence in China also increased rapidly from $0.67 \%$ in 1980 to $10.4 \%$ in 2013 (3). Therefore, DM is a contributing factor to morbidity and mortality. So far, various organizations have developed various diabetic guidelines to clarify the definition, classification, diagnosis, screening, and prevention of this disease, which are used not for clinical management but also the monitoring of ongoing care with laboratory check-ups at regular intervals, lifestyle counseling, and prevention of diabetic-related complications.

The diagnostic criteria for DM are based primarily on fasting plasma glucose (FPG), random plasma glucose or oral glucose tolerance test (OGTT) 2-hour plasma glucose (2hPG). In 2011, WHO recommended wherever conditions permit, countries and regions may consider adopting the hemoglobin A1c (HbA1c) $\geq 6.5 \%$ as the cut-point for a diabetes diagnosis. Several studies have shown that the optimal cut-off value for HbA1c to diagnose diabetes in Chinese adults is $6.3 \%$. However, HbA1c has not yet been included in the latest guidelines for diabetes in China (3).

DM is classified into four types: type 1 diabetes mellitus (T1DM), T2DM, other specific types of diabetes, and gestational diabetes mellitus (GDM), whereas T2DM is the most common form and occurs when the target tissue (the liver, skeletal muscles, and adipose tissues) loses insulin sensitivity. In the United States, the American Diabetes 
Association (ADA) and the European Association for the Study of Diabetes (EASD) guidelines, and the American Association of Clinical Endocrinologists (AACE) and the American College of Endocrinology (ACE) guidelines are two typically consulted to determine optimal therapeutic decisions in patients with T2DM. The differences between the two guidelines are: (I) the ADA/EASD guideline advocates a stepwise approach to glycemic control, starting with metformin and incrementally intensifying to dual and triple therapy at 3-month intervals until the patient is at their individualized goal, whereas the AACE/ACE guideline provides a broader choice of first-line medications, with a suggested hierarchy of use. It encourages initial dual and triple therapy if the HbA1c level is $7.59 .0 \%$ and $>9.0 \%$ at diagnosis, respectively. (II) Compared with the value in the AACE/ACE guideline, the target HbA1c value is higher in the ADA/EASD guideline ( $\leq 6.5 \%$ vs. $\leq 7.0 \%)$ (4). Considering the ethnic characteristics, social culture, and social resources, the Chinese Diabetes Society (CDS) has drawn up the latest version of guidelines for the prevention and care of T2DM in China in 2019, which may work best for the Chinese patient population. This guideline recommends the target $\mathrm{HbA1c}$ value, which is $<7 \%$ for most nonpregnant adults with T2DM, and drug therapy should be started when HbAlc value is $\geq 7.0 \%$. Metformin, $\alpha$-glucosidase inhibitors, or insulin secretagogues could be options for monotherapy. If monotherapy is insufficient, dual and triple therapy or multiple daily insulin injections may be prescribed (3). Diabetes is a complicated disease that affects multiple organs, requiring multiple treatments and preventive strategies to prevent long-term complications associated with it. The following is a brief narrative review of the diagnosis, prevention and treatment of diabetic complications. MEDLINE was searched using the terms "diabetic nephropathy", "diabetes and dyslipidemia", "Apolipoprotein M", "diabetes and obstructive sleep apnea" for nearly five years.

We present the following article in accordance with the Narrative Review reporting checklist (available at http:// dx.doi.org/10.21037/atm-20-7243).

\section{Diabetic nephropathy (DN)}

$\mathrm{DN}$, as one of the most prevalent lethal complications of diabetes, is observed in approximately $20 \%$ to $30 \%$ of T2DM individuals (5). The etiology of $\mathrm{DN}$ is multifactor and involves many molecular signaling pathway abnormalities, including the advanced glycation end products/receptors for AGE (AGE/RAGE), protein kinase $\mathrm{C}$ (PKC), reactive oxygen species (ROS), mammalian target of rapamycin (mTOR), Janus Kinase/Signal Transducer and Activator of Transcription Proteins (JAK/STAT), etc. (6). Chronic hypoxia is one feature of DN. At present, the clinical diagnosis of DN mainly depends on the elevated urinary albumin excretion and reduced eGFR in the absence of other primary causes of kidney damage, lacking high sensitivity and specificity indicators to reflect the renal blood oxygen levels. A growing number of studies have focused on the biomarkers for early diagnosis of $\mathrm{DN}$, which include (I) certain urinary microRNAs such as microRNA-210 and microRNA-34a (7), urinary kidney injury molecule 1 (KIM-1) and Chitinase-3-like protein 1 (YKL-40) (8), urinary E-cadherin (9); (II) serum galectin-3 and growth differentiation factor-15 (GDF-15) (10), serum pregnenolone sulfate, ganglioside GA1, phosphatidyl glycerol and all-trans-Carophyll yellow tested by direct flow through mass spectrometry (11); (III) suppressor of mothers against decapentaplegic type 1 (SMAD1) and neutrophil gelatinase-associated lipocalin (NGAL) gene expression in peripheral blood and urine samples (12). However, renal biopsy is still the gold standard to diagnose diabetic nephropathy. Tong et al. have searched identified 40 studies worldwide between 1977 and 2019 that looked at global renal biopsy and pathological nondiabetic kidney disease (NDKD) lesions. The results have shown that overall prevalence rate of DN, NDKD and DN plus NDKD is $41.3,40.6$ and $18.1 \%$, respectively (13). Therefore, it is of great significance to do renal biopsy as early as possible to confirm the diagnosis and to enable personalized treatment especially when T2DM patients present atypical diabetic kidney disease (DKD) symptoms (e.g., absence of diabetic retinopathy, shorter duration of diabetes, microscopic hematuria, subnephrotic range proteinuria, lower glycated hemoglobin, lower fasting blood glucose). Ding et al. have shown that texture analysis with DWI, BOLD, and susceptibility-weighted imaging (SWI) can help assess renal dysfunction during the early stages of chronic kidney disease (14). Jiang et al. reported the role of SWI parameters and SWI-based texture features in evaluating renal dysfunction of T2DM (15). They showed that the signal intensity ratio of the medulla to psoas muscle (MPswi) was significantly lower than the signal intensity ratio of the cortex to psoas muscle (CPswi) in non-moderate-severe renal injured (non-msRI) group and msRI group. MPswi was higher, whereas the signal intensity ratio of the cortex to the medulla (CMswi), skewness, the correlation was lower 
in the msRI group than in non-msRI group and CMswi was an independent protective factor for msRI. These results supply a crucial noninvasive method to monitor renal blood oxygen levels, which can help understand the kidney oxygenation status and fibrosis process and monitor the efficacy of new drugs for DN. As a noninvasive imaging diagnostic method, MRI provides some level of spatial resolution and it can be performed repeatedly, providing some level of temporal resolution. Moreover, it is noninvasive and thus can be applied to both animals used in experiments and humans, providing a pathway for translation of new therapies. So far, blood oxygen leveldependent (BOLD) MRI, diffusion tensor imaging (DTI) MRI and dynamic nuclear polarization (DNP) MRI have been confirmed to be used for early identification of DN. A major advantage of BOLD-MRI is that it does not require an exogenous paramagnetic agent, because it relies on the differing paramagnetic properties of oxyhemoglobin and deoxyhemoglobin. BOLD imaging detected the medullary hypoxia at the simply diabetic stage, while DTI didn't identify the medullary directional diffusion changes at this stage. Therefore, BOLD imaging may be more sensitive for assessment of the early renal function changes than DTI (16). Furthermore, BOLD-MRI, coupled with other imaging modalities that provide information about renal hemodynamics and function, has provided important insights into the temporal and spatial relationships between renal hypoxia and progression of diabetic nephropathy (17). However, there are several disadvantages of BOLD-MRI. First, BOLD-MRI provides a measure of blood oxygenation rather than tissue oxygenation. Second, it is not quantitative in the sense that it cannot provide an actual level of $\mathrm{PO}_{2}$ or even of blood hemoglobin saturation. Third, it is susceptible to artefacts caused by changes in renal blood volume and hemoglobin concentration. DNP-MRI combined with the oxygen-sensitive paramagnetic agent OX63 (18) is a method for quantifying tissue oxygen tension within the kidney. Nevertheless, this method needs to use an exogenous paramagnetic agent that must be administered intravenously and OX63 is also rather expensive. Therefore, it is used only in small animals such as the mice. Furthermore, either general anesthesia or physical restraint is required during imaging.

Although several clinical approaches are available to relieve symptoms temporarily or impede the progression of $\mathrm{DN}$, there is no curative treatment to date. Therefore, a novel therapeutic strategy is warranted to control DN. MicroRNAs (miRNAs) are a class of non-coding RNAs that can bind to their target mRNAs to take part in the epigenetic machinery of their downstream signaling molecules. Over the past few decades, a plethora of studies has revealed the potential involvement of different miRNAs in different molecular and signaling pathways leading to DN. Several reviews and studies have shown in-depth the vital role of various miRNAs in progression, diagnosis, and therapeutics of DN (19). Concretely speaking, several miRNAs including miR-21, miR-124, miR-135a, miR192, miR-195, miR-200, miR-215, miR-216a, miR217, miR-377, and miR-1207-5p, are overexpressed in DN, whereas some miRNAs (Let-7, miR-25, miR-29, miR-93, miR-141, miR-200, and miR-451) are downregulated (20). The roles of miRNAs are multifaceted, involved in the TGF- $\beta$ signaling pathway, in the PI3K/Akt signaling pathway, in collagen gene expression, in DNA methyltransferases (DNMTs) and histone deacetylases (HDACs), in epithelial-to-mesenchymal transition (EMT) and endothelial-mesenchymal transition (EndMT) and so on (20). Therefore, both up- and down-regulation of $\mathrm{DN}$-suppressing and $\mathrm{DN}$-promoting miRNAs can be exploited as therapeutic interventions in DN, respectively. Pharmacological inhibition of miRNAs can be achieved through employing miRNA inhibitors, anti-miRNA oligonucleotides (AMOs) and miRNA sponges, while upregulation of miRNAs can be accomplished using miRNA mimics, miRNA-containing exosomes and miRNA promoters (20).

Except for the microRNAs (miRNAs), long noncoding RNAs (lncRNAs) and circular RNAs (circRNAs) are also play an important role in $\mathrm{DN}$ progression. Several lncRNAs such as the Plasmacytoma Variant Translocation-1 (PVT1) LncRNA, the Nuclear Enriched Abundant Transcript-1 (NEAT1) LncRNA, LncRNA ERBB4-IR, LncRNA CYP4B1-PS1-001 and LncRNA ENSMUST00000147869 are involved in the regulation of renal hypertrophy and extracellular matrix (ECM) accumulation. A few IncRNAs such as LncRNA GM4419, the Metastasisassociated lung adenocarcinoma transcript-1 (MALAT-1) LncRNA, LncRNA GM6135, myocardial infarctionassociated transcript (MIAT) LncRNA can regulate renal inflammation and oxidative stress. Furthermore, LncRNA GM5524, LncRNA GAS5, LncRNA GM15645, the Taurine upregulated-1 (TUG-1) LncRNA and the Cancer susceptibility candidate 2 (CASC2) LncRNA are involved in the renal autophagy and apoptosis (21). The evidence to elucidate the interaction of circular RNA in DN progression is limited. Only one study in DN mice showed 
that circRNA_15698 expression could regulate the fibrosis of mesangial cells (22).

Connexins (Cxs) are a multigenic family of transmembrane proteins that form gap junction membrane channels and take part in the exchange of information between adjacent cells. Cx43 is the most abundantly expressed and widely distributed gap junction protein. A previous study has shown that the expression of $\mathrm{Cx} 43$ was significantly decreased in DN patients and diabetic model animals (23). Other studies have shown that the upregulation of $\mathrm{Cx} 43$ was involved in podocyte injury, and the interdependence of $\mathrm{Cx} 43$ and impaired autophagic flux may be a novel mechanism of podocyte injury in DN $(24,25)$. Also, one latest research has shown the essential role of $\mathrm{Cx} 43$ in regulating renal EMT and diabetic renal tubulointerstitial fibrosis via regulating the SIRT1-HIF-1 $\alpha$ signaling pathway and provided an experimental basis for Cx43 as a potential target for DN (26).

Li et al. put forward a new viewpoint that there was a link between miRNA-30 and Cx43 in the podocyte under high glucose ambiance (HG) or diabetic state (27). They used streptozotocin (STZ)-induced rat model of diabetes and podocyte culture under HG. Both the podocytes and rats were concomitantly treated with miRNA-30 mimic or miRNA-30 inhibitors. The results showed that the expression of miRNA-30 was down-regulated, and the transfection of miRNA-30 reduced podocyte injury, apoptosis, and ERS, both in vivo and in vitro. Luciferase reporter assays confirmed $\mathrm{Cx} 43$ is a directed target of microRNA-30s. Likewise, Cx43 siRNA treatment yielded comparable results. From these results, the authors concluded that the overexpression of miRNA-30 prevents DN-induced podocyte damage by modulating $\mathrm{Cx} 43$ expression. This study provides novel information that highlights the microRNA-30/Cx43/ ERS axis, plays a role in the pathogenesis of $\mathrm{DN}$, and it may serve as a potential therapeutic target for the amelioration of DN. However, considerable research is needed for a better understanding of the regulation and functions of this signaling pathway in DN.

\section{Diabetes and dyslipidemia}

Glucose intolerance, dyslipidemia, combined with abdominal obesity and hypertension, together constitute the metabolic syndrome (MetS), which results in a significant increase in morbidity and all-cause mortality. A 10-year prospective study has shown that MetS could lead to 1.80 and 2.05 times higher statistically significant probability for myocardial infarction (MI) and stroke events, respectively. Further studies have shown that abdominal obesity increases the risk of MI, and low levels of high-density lipoprotein cholesterol (HDL-C) and hypertriglyceridemia increase the risk of stroke in men with MetS (28). Dyslipidemia in adolescence is usually associated with one or more components of the MetS, namely obesity, hypertension, and impaired glucose tolerance, and presents with high triglyceride and low HDL-C. In one trial of adolescents with T2DM, 21\% had high triglyceride levels or had been treated with lipid-lowering drugs at baseline, and 23\% had high triglyceride (TG) levels three years later (29). It is worth noting that there is increasing evidence linking T2DM to atherosclerosis, which may attribute to local activation of the RAS and its components, including receptors and relevant enzymes in the microvessel (30). Also, high TG, low HDL-C, increased LDL particle number or apolipoprotein B, smaller LDL size and density, and LDL glycation and oxidation are all associated with increased atherosclerosis (31). The EVAPORATE trial has revealed HDL-C levels are negatively correlated with baseline coronary plaque, total plaque (TP), and total noncalcified plaque (TNCP) volumes, providing more detailed mechanistic evidence for the protective effect of HDL-C on coronary atherosclerosis in high-risk populations (32). China's latest guideline has recommended that the therapeutic targets for lipids are: LDL-C $<2.60 \mathrm{mmol} / \mathrm{L}$, TG $<1.70 \mathrm{mmol} / \mathrm{L}, \mathrm{HDL}-\mathrm{C} \geq 1.0 \mathrm{mmol} / \mathrm{L}$, to prevent clinical cardiovascular disease (CVD) (3). Therefore, treatment measures should be taken when LDL-C, TG, and HDL-C levels exceed cut points in diabetic patients.

Apolipoprotein $M$ (apoM) is a novel apolipoprotein bound primarily to HDL in plasma. Many previous studies have shown that in diabetic mouse models, the expression of apoM in plasma, liver, and kidney is all remarkably decreased, which may be attributed to the decreased expression of peroxisome proliferator-activated receptor and liver X receptor secondary to hyperglycemia $(33,34)$. However, the results of studies on apoM expression in diabetic patients are inconsistent. Several studies have shown that plasma apoM levels decreased significantly in maturityonset diabetes of the young 3 (MODY3) individuals with HNF-1 mutation, whereas these levels remained unchanged in MODY1 patients (35). Contradicting results have been reported no significant differences in apoM concentrations among MODY3, T2DM, and healthy individuals (36). The inconsistency between studies may apply to differences 
in methods (e.g., dot blot, immunoblot, and ELISA) used to detect serum apoM. Also, previous studies have determined apoM levels were negatively correlated with the development and progression of diabetes, which could be attributed to Sphingosine-1-Phosphate, a functional lipid that can promote $\beta$-cell functions, and insulin secretion and improve glucose tolerance $(37,38)$. Finally, genetic variations in the apo $M$ gene have also played a prominent role in diabetes susceptibility. Wu et al. found that SNP T-778C was strongly associated with T1DM in both Han Chinese and Swedish populations, and allele C of SNP T-778C may increase promoter activity and confer the risk susceptibility to the development of T1DM (39). More recently, a study by Liu et al. suggested that rs805296 (T-778C)-C and rs9404941 (T-855C)-C alleles haplotypes were indicators of high T2DM risk (40). However, another study drew a different conclusion that there was no association between the apoM gene and T2DM susceptibility in Hong Kong Chinese cohort. Interestingly, the C allele of rs805297 was significantly associated with T2DM duration of longer than ten years (41). Therefore, the relationship between apoM and diabetes deserves further investigation.

According to the study by Yao et al. (42), apoM is expressed on all peripheral blood mononuclear cells (PBMCs). Impressively, compared with the other monocytes, $\mathrm{CD} 14^{+}$cells, the central immune cells, in the atherosclerotic process, have the highest rates of apoM+ cells, suggesting that apoM might take part in the pathological lesions of atherosclerosis. The investigators found both apoM $\mathrm{M}^{+} \mathrm{HDL}$ and apoM- $\mathrm{MDL}^{-}$could downregulate the expression of IL-6, IL-1 $\beta$, and MCP-1 in macrophages, and the downregulation of TNF- $\alpha$, IL-6, and IL- $1 \beta$ was more significant in the apo $M^{+}$HDL group than in the apoM $\mathrm{M}^{-}$HDL group. Notably, the expression of IL-10, an anti-inflammatory cytokine preventing lipid deposition and inflammation in atherosclerotic lesions, was significantly upregulated in the apo $\mathrm{M}^{+} \mathrm{HDL}$ group. In this study, the investigators used coimmunoprecipitation to detect the binding of purified apo $\mathrm{M}^{+} \mathrm{HDL}$ and apoM ${ }^{-}$ HDL proteins to SR-BI, the physiological receptor for apoA-I/HDL which was expressed on the surface of THP1 macrophages. The results showed that the recombinant human apoM, human apo $\mathrm{M}^{-} \mathrm{HDL}$, and human apoM $\mathrm{M}^{+}$ HDL particles could interact with SR-BI, highlighting that apoM could promote the anti-inflammatory effects of HDL, possibly by binding to SR-BI. However, further studies are necessary to investigate the downstream signaling pathway.

\section{Diabetes and obstructive sleep apnea (OSA)}

OSA is characterized by recurrent partial (hypopnea) or complete (apnea) upper airway obstruction, leading to hypoxia, recurrent arousal from sleep, and desaturationreoxygenation sequences (43). Data from previous research suggest OSA is associated with many metabolic abnormalities, including impaired fatty acid handling, glucose tolerance, and insulin sensitivity, atherogenesis, and high blood pressure, through the effects of sleep fragmentation, intermittent hypoxia, sympathetic overactivity, and adipose tissue inflammation $(43,44)$. Also, many studies have confirmed that OSA is an independent risk factor for CVD (45). Notably, a recent study showed that OSA could cause a significant increase in all-cause mortality and major adverse cardiac events (MACEs) in patients with T2DM and co-existing OSA following percutaneous coronary intervention (PCI) (46). The investigators proposed several mechanisms by which OSA contributes to cardiovascular events, including endothelial dysfunction, vascular inflammation, and high platelet reactions resulting from intermittent hypoxemia, autonomic imbalance, and sleep disruption (47). Therefore, early diagnosis and management of OSA might be critical for patients with T2DM to reduce the risk of MACEs.

A study by Shi et al. (48) constructed and validated an easy-to-use nomogram comprising six items, namely waistto-hip ratio, smoking status, body mass index, serum uric acid, HOMA-IR and history of fatty liver which could accurately predict and rapidly assess the risk of OSA in patients with T2DM, and help identify patients at high risk of OSA that should be referred for diagnostic polysomnography. This nomogram, as a lesscostly and timeconsuming examination, is worthy of clinical promotion to reduce the number of missed OSA diagnoses in patients with T2DM. However, the sensitivity and specificity of this nomogram need further evaluation in the general population.

\section{Conclusions}

Diabetes is a metabolic disorder that has resulted in critical personal health hazards and public severe health burdens worldwide, often accompanied by chronic systemic complications in multiple organs and systems, including diabetic nephropathy, diabetic retinopathy, diabetic neuropathy, and so on. Moreover, diabetes is associated with metabolic syndrome and OSA. Simple and easy 
detection methods with high sensitivity are conducive to the early diagnosis of diabetic complications. Clarifying the mechanism of diabetic complications is conducive to develop new drugs and therapies.

\section{Acknowledgments}

Funding: None

\section{Footnote}

Reporting Checklist: The author was completed the Narrative Review reporting checklist. Available at http://dx.doi. org/10.21037/atm-20-7243

Conflicts of Interest: The author has completed the ICMJE uniform disclosure form (available at http://dx.doi. org/10.21037/atm-20-7243). The author has no conflicts of interest to declare.

Etbical Statement: The author is accountable for all aspects of the work in ensuring that questions related to the accuracy or integrity of any part of the work are appropriately investigated and resolved.

Open Access Statement: This is an Open Access article distributed in accordance with the Creative Commons Attribution-NonCommercial-NoDerivs 4.0 International License (CC BY-NC-ND 4.0), which permits the noncommercial replication and distribution of the article with the strict proviso that no changes or edits are made and the original work is properly cited (including links to both the formal publication through the relevant DOI and the license). See: https://creativecommons.org/licenses/by-nc-nd/4.0/.

\section{References}

1. AghaeiZarch SM, DehghanTezerjani M, Talebi M, et al. Molecular biomarkers in diabetes mellitus (DM). Med J Islam Repub Iran 2020;34:28.

2. Hashmi NR, Khan SA. Adherence To Diabetes Mellitus Treatment Guidelines From Theory To Practice: The Missing Link. J Ayub Med Coll Abbottabad 2016;28:802-8.

3. Jia W, Weng J, Zhu D, et al. Standards of medical care for type 2 diabetes in China 2019. Diabetes Metab Res Rev 2019;35:e3158.

4. Cornell S. Comparison of the diabetes guidelines from the ADA/EASD and the AACE/ACE. J Am Pharm Assoc
(2003) 2017;57:261-5.

5. Holt RIG. Lest we forget the microvascular complications of diabetes. Diabet Med 2018;35:1307.

6. Meza Letelier CE, San Martín Ojeda CA, Ruiz Provoste JJ, et al. Pathophysiology of diabetic nephropathy: a literature review. Medwave 2017;17:e6839.

7. Nossier AI, Shehata NI, Morsy SM, et al. Determination of certain urinary microRNAs as promising biomarkers in diabetic nephropathy patients using gold nanoparticles. Anal Biochem 2020;609:113967.

8. Kapoula GV, Kontou PI, Bagos PG. Diagnostic Performance of Biomarkers Urinary KIM-1 and YKL40 for Early Diabetic Nephropathy, in Patients with Type 2 Diabetes: A Systematic Review and Meta-Analysis. Diagnostics (Basel) 2020;10:909.

9. Koziolek M, Mueller GA, Dihazi GH, et al. Urine E-cadherin: A Marker for Early Detection of Kidney Injury in Diabetic Patients. J Clin Med 2020;9:639.

10. Hussain S, Habib A, Hussain MS, et al. Potential biomarkers for early detection of diabetic kidney disease. Diabetes Res Clin Pract 2020;161:108082.

11. Nilavan E, Sundar S, Shenbagamoorthy M, et al. Identification of biomarkers for early diagnosis of diabetic nephropathy disease using direct flow through mass spectrometry. Diabetes Metab Syndr 2020;14:2073-8.

12. Veiga G, Alves B, Perez M, et al. NGAL and SMAD1 gene expression in the early detection of diabetic nephropathy by liquid biopsy. J Clin Pathol 2020;73:713-21.

13. Tong X, Yu Q, Ankawi G, et al. Insights into the Role of Renal Biopsy in Patients with T2DM: A Literature Review of Global Renal Biopsy Results. Diabetes Ther 2020;11:1983-99.

14. Ding J, Xing Z, Jiang Z, et al. Evaluation of renal dysfunction using texture analysis based on DWI, BOLD, and susceptibility-weighted imaging. Eur Radiol 2019;29:2293-301.

15. Jiang Z, Wang Y, Ding J, et al. Susceptibility weighted imaging (SWI) for evaluating renal dysfunction in type 2 diabetes mellitus: a preliminary study using SWI parameters and SWI-based texture features. Ann Transl Med 2020;8:1673.

16. Feng YZ, Ye YJ, Cheng ZY, et al. Non-invasive assessment of early stage diabetic nephropathy by DTI and BOLD MRI. Br J Radiol 2020;93:20190562.

17. Evans RG. Another step forward for methods for studying renal oxygenation. Kidney Int 2019;96:552-4.

18. Kodama Y, Hyodo F, Yamato M, et al. Dynamic nuclear polarization magnetic resonance imaging and the oxygen- 
sensitive paramagnetic agent $\mathrm{OX} 63$ provide a noninvasive quantitative evaluation of kidney hypoxia in diabetic mice. Kidney Int 2019;96:787-92.

19. Sankrityayan H, Kulkarni YA, Gaikwad AB. Diabetic nephropathy: The regulatory interplay between epigenetics and microRNAs. Pharmacol Res 2019;141:574-85.

20. Dewanjee $S$, Bhattacharjee N. MicroRNA: A new generation therapeutic target in diabetic nephropathy. Biochem Pharmacol 2018;155:32-47.

21. Loganathan TS, Sulaiman SA, Abdul Murad NA, et al. Interactions Among Non-Coding RNAs in Diabetic Nephropathy. Front Pharmacol 2020;11:191.

22. Hu W, Han Q, Zhao L, et al. Circular RNA circRNA_15698 aggravates the extracellular matrix of diabetic nephropathy mesangial cells via miR-185/ TGF- $\beta 1$. J Cell Physiol 2019;234:1469-76.

23. Zeng O, Li F, Li Y, et al. Effect of Novel Gasotransmitter hydrogen sulfide on renal fibrosis and connexins expression in diabetic rats. Bioengineered 2016;7:314-20.

24. Yang M, Wang B, Li M, et al. Connexin 43 is involved in aldosterone-induced podocyte injury. Cell Physiol Biochem 2014;34:1652-62.

25. Ji J, Zhao Y, Na C, et al. Connexin 43-autophagy loop in the podocyte injury of diabetic nephropathy. Int J Mol Med 2019;44:1781-8.

26. Sun X, Huang K, Haiming X, et al. Connexin 43 prevents the progression of diabetic renal tubulointerstitial fibrosis by regulating the SIRT1-HIF-1 $\alpha$ signaling pathway. Clin Sci (Lond) 2020;134:1573-92.

27. Li M, Ni W, Zhang M, et al. MicroRNA-30/Cx43 axis contributes to podocyte injury by regulating ER stress in diabetic nephropathy. Ann Transl Med 2020;8:1674.

28. Kazlauskienè L, Butnorienè J, Norkus A. Metabolic syndrome related to cardiovascular events in a 10-year prospective study. Diabetol Metab Syndr 2015;7:102.

29. Lipid and inflammatory cardiovascular risk worsens over 3 years in youth with type 2 diabetes: the TODAY clinical trial. Diabetes Care 2013;36:1758-64.

30. La Sala L, Prattichizzo F, Ceriello A. The link between diabetes and atherosclerosis. Eur J Prev Cardiol 2019;26:15-24.

31. Bergheanu SC, Bodde MC, Jukema JW. Pathophysiology and treatment of atherosclerosis : Current view and future perspective on lipoprotein modification treatment. Neth Heart J 2017;25:231-42.

32. Lakshmanan S, Shekar C, Kinninger A, et al. Association of high-density lipoprotein levels with baseline coronary plaque volumes by coronary CTA in the EVAPORATE trial. Atherosclerosis 2020;305:34-41.

33. Zhang X, Jiang B, Luo G, et al. Hyperglycemia downregulates apolipoprotein $\mathrm{M}$ expression in vivo and in vitro. Biochim Biophys Acta 2007;1771:879-82.

34. Luo G, Feng Y, Zhang J, et al. Rosiglitazone enhances apolipoprotein M (Apom) expression in rat's liver. Int J Med Sci 2014;11:1015-21.

35. Richter S, Shih DQ, Pearson ER, et al. Regulation of apolipoprotein $\mathrm{M}$ gene expression by MODY3 gene hepatocyte nuclear factor-1alpha: haploinsufficiency is associated with reduced serum apolipoprotein $M$ levels. Diabetes 2003;52:2989-95.

36. Cervin C, Axler O, Holmkvist J, et al. An investigation of serum concentration of apoM as a potential MODY3 marker using a novel ELISA. J Intern Med 2010;267:316-21.

37. Qi Y, Chen J, Lay A, et al. Loss of sphingosine kinase 1 predisposes to the onset of diabetes via promoting pancreatic $\beta$-cell death in diet-induced obese mice. FASEB J 2013;27:4294-304.

38. Wang J, Badeanlou L, Bielawski J, et al. Sphingosine kinase 1 regulates adipose proinflammatory responses and insulin resistance. Am J Physiol Endocrinol Metab 2014;306:E756-68.

39. Wu X, Niu N, Brismar K, et al. Apolipoprotein M promoter polymorphisms alter promoter activity and confer the susceptibility to the development of type 1 diabetes. Clin Biochem 2009;42:17-21.

40. Liu D, Pan JM, Pei X, et al. Interaction Between Apolipoprotein M Gene Single-Nucleotide Polymorphisms and Obesity and its Effect on Type 2 Diabetes Mellitus Susceptibility. Sci Rep 2020;10:7859.

41. Zhou JW, Tsui SK, Ng MC, et al. Apolipoprotein M gene (APOM) polymorphism modifies metabolic and disease traits in type 2 diabetes. PLoS One 2011;6:e17324.

42. Yao S, Luo G, Liu H,et al. Apolipoprotein M promotes the anti-inflammatory effect of high-density lipoprotein by binding to scavenger receptor BI. Ann Transl Med 2020;8:1676.

43. Kamble PG, Theorell-Haglöw J, Wiklund U, et al. Sleep apnea in men is associated with altered lipid metabolism, glucose tolerance, insulin sensitivity, and body fat percentage. Endocrine 2020;70:48-57.

44. Ryan S. Adipose tissue inflammation by intermittent hypoxia: mechanistic link between obstructive sleep apnoea and metabolic dysfunction. J Physiol 2017;595:2423-30.

45. Mazzotti DR, Keenan BT, Lim DC, et al. Symptom Subtypes of Obstructive Sleep Apnea Predict Incidence 
Page 8 of 8

of Cardiovascular Outcomes. Am J Respir Crit Care Med 2019;200:493-506.

46. Wang H, Li X, Tang Z, et al. Cardiovascular Outcomes Post Percutaneous Coronary Intervention in Patients with Obstructive Sleep Apnea and Type 2 Diabetes Mellitus: A Systematic Review and Meta-Analysis. Diabetes Ther 2020;11:1795-806.

47. Karakaş MS, Altekin RE, Baktır AO, et al. Association between mean platelet volume and severity of disease in patients with obstructive sleep apnea syndrome without risk factors for cardiovascular disease. Turk Kardiyol Dern Ars 2013;41:14-20.

48. Shi H, Xiang S, Huang X, et al. Development and validation of a nomogram for predicting the risk of obstructive sleep apnea in patients with type 2 diabetes. Ann Transl Med 2020;8:1675.

(English Language Editor: J. Chapnick)

Cite this article as: Hua F. New insights into diabetes mellitus and its complications: a narrative review. Ann Transl Med 2020;8(24):1689. doi: 10.21037/atm-20-7243 